\title{
Construction and analysis of the IncRNA-miRNA-mRNA network based on competitive endogenous RNA reveals functional genes in heart failure
}

\author{
GUOHONG WANG ${ }^{1}$, XIANGHUI ZHENG ${ }^{2,3}$, YANG ZHENG $^{2,3}$, RUI CAO $^{4}$, \\ MAOMAO ZHANG ${ }^{2,3}$, YONG SUN ${ }^{2,3}$ and JIAN WU $\mathrm{W}^{2,3}$
}

\author{
${ }^{1}$ Department of Cardiovascular Center of Beijing Tongren Hospital, Affiliated to Capital Medical University, \\ Beijing 100730; ${ }^{2}$ Department of Cardiology, The Second Affiliated Hospital of Harbin Medical University; \\ ${ }^{3}$ The Key Laboratory of Myocardial Ischemia, Harbin Medical University, Ministry of Education; \\ ${ }^{4}$ Cardiovascular Department, The First Hospital of Harbin, Harbin, Heilongjiang 150010, P.R. China
}

Received April 22, 2018; Accepted November 19, 2018

DOI: $10.3892 / \mathrm{mmr} .2018 .9734$

\begin{abstract}
Heart failure (HF) is a principal cause of morbidity and mortality worldwide, affecting an estimated 38 million people. Although significant progress has been made with respect to the underlying molecular mechanisms, the role of the competing endogenous RNA (ceRNA) network in the pathogenesis of HF remains largely unknown. In this study, an HF-associated ceRNA network was constructed based on the differentially expressed long noncoding RNAs (lncRNAs), microRNAs (miRNAs) and mRNAs obtained, respectively, from the GSE77399, GSE104150 and GSE84796 datasets. The ceRNA network consisted of 12 lncRNA nodes, 43 miRNA nodes, 343 mRNA nodes and 530 edges. Gene ontology and Kyoto Encyclopedia of Genes and Genomes pathway analysis demonstrated that the ceRNA network was primarily enriched in the immune response, inflammatory response and T cell and $B$ cell receptor signaling pathways. In addition, three lncRNAs (growth arrest specific 5, taurine upregulated 1 and HOX transcript antisense RNA) and three miRNAs [hsa-miRNA (miR)-26b-5p, hsa-miR-8485 and hsa-miR-940] with higher node degrees compared with other genes were selected as hub nodes. The expression of hub nodes in patients with HF was verified by reverse transcription-quantitative polymerase chain
\end{abstract}

Correspondence to: Professor Jian Wu, Department of Cardiology, The Second Affiliated Hospital of Harbin Medical University, 246 Xuefu Road, Nangang, Harbin, Heilongjiang 150010, P.R. China E-mail:wujian780805@163.com

Abbreviations: HF, heart failure; lncRNAs, long noncoding RNAs; miRNAs, microRNAs; ceRNA, competing endogenous RNA; GEO, Gene Expression Omnibus; DELs, differentially expressed lncRNAs; DEMis, differentially expressed miRNAs; DEMs, differentially expressed mRNAs; GO, gene ontology; KEGG, Kyoto Encyclopedia of Genes and Genomes

Key words: heart failure, miRNA, long noncoding RNA, competing endogenous RNA reaction analysis. The present study provided further insights into the important roles of the ceRNA network in HF development, and indicated the potential use of these hub nodes as diagnostic biomarkers and therapeutic targets.

\section{Introduction}

Heart failure (HF) is a global public health concern with an estimated prevalence of 38 million patients worldwide (1). $\mathrm{HF}$ is the end stage of various vascular diseases, including hypertension, valvular heart disease and myocardial infarction (2), and is commonly caused by ischemic heart disease (3). The heart responds to stress or cardiac insults via a compensatory increase in size and mass, termed cardiac hypertrophy. With the development of heart disease, the structure and metabolism of the heart alters to maintain cardiac function. However, due to the chronic accompanying stress, the ventricles dilate and the condition progresses to HF $(4,5)$. Previous studies have provided novel insights into the pathobiology of $\mathrm{HF}$ and the underlying molecular mechanisms $(6,7)$. However, the precise molecular etiology remains unclear, and thus requires investigation in order to identify novel therapeutic targets.

Studies have demonstrated that noncoding RNAs serve crucial roles in numerous diseases (8-10). They are classified into two classes: Small noncoding RNAs [microRNAs (miRNAs), small interfering RNAs and transfer RNAs] and long noncoding RNAs (lncRNAs). miRNAs and lncRNAs regulate each other in various signaling pathways and biological processes. Salmena et al (11) proposed the competing endogenous RNA (ceRNA) hypothesis, wherein lncRNAs harboring miRNA response elements compete with one another to bind to a common miRNA, and thereby act as molecular 'sponges' and de-repress the target genes of the miRNAs (11). A ceRNA network has been demonstrated in numerous diseases, particularly in cancer. Chen et al (12) identified an upregulation of the lncRNA long intergenic non-protein coding RNA 1234 (LINC01234) axis in gastric cancer tissues, wherein LINC01234 acted as the ceRNA for 
miR-204-5p and modulated its endogenous target core-binding factor subunit $\beta$. However, no ceRNA networks have been associated with HF thus far.

In the present study, a ceRNA network was constructed using data from the GSE77399, GSE104150 and GSE84796 datasets downloaded from the Gene Expression Omnibus (GEO) database, to identify differentially expressed lncRNAs (DELs), miRNAs (DEMis) and mRNAs (DEMs) using GEO2R. Subsequently, target lncRNAs and mRNAs of the miRNAs were predicted using the appropriate databases, and the ceRNA network was constructed and analyzed using Cytoscape. A total of six hub nodes were identified, including hsa-miR-26b-5p, hsa-miR-8485, hsa-miR-940, growth arrest specific 5 (GAS5), taurine upregulated 1 (TUG1) and HOX transcript antisense RNA (Hotair). The expression levels of hsa-miR-940, GAS5 and Hotair were increased and those of hsa-miR-26-5p, hsa-miR-8485 and TUG1 were decreased in patients with HF. The present study may not only provide a novel regulatory mechanism between noncoding and coding RNAs in HF, but also deepen the understanding of its development and progression.

\section{Materials and methods}

Data collection. The GEO (https://www.ncbi.nlm.nih. gov/geo/) is an international, public functional genomics data repository for high-throughput microarray and next-generation sequences (13). The recently published gene expression profiles related to HF (GSE77399, GSE104150 and GSE84796) were downloaded from GEO (http://www. ncbi.nlm.nih.gov/geo). The GSE77399 IncRNA dataset was measured using GPL21384 Platforms (Human Disease-related LncRNA Profiler, System Biosciences, California, USA), and included data from 13 patients with HF and 12 healthy controls. The miRNA expression data in GSE104150 were based on GPL20712 Platforms (Agilent-070156 Human miRNA, miRNA version; Agilent Technologies, Inc., Santa Clara, CA, USA), and consisted of data from nine patients with $\mathrm{HF}$ and seven healthy controls. The mRNA expression data in GSE84796 were from 10 patients with HF and seven healthy controls, and were based on GPL14550 (Agilent-028004 SurePrint G3 Human GE 8x60 K Microarray, Probe Name Version; Agilent Technologies, Inc.).

Screening of differentially expressed lncRNAs, miRNAs and mRNAs. The DELs, DEMis and DEMs between the HF patients and healthy controls were analyzed by GEO2R (https://www.ncbi.nlm.nih.gov/geo/geo2r/). The false discovery rate (FDR) is a method of conceptualizing the rate of type I errors in null hypothesis testing when conducting multiple comparisons (14). GEO2R is able to calculate the FDR automatically. The screening thresholds were FDR corrected $\mathrm{P}<0.05$ andllog2(fold-change) $\mid>1$.

Prediction of target lncRNAs and mRNAs of miRNAs. The lncRNA targets of the miRNAs were predicted using DIANA-LncBase v2 (www.microrna.gr/LncBase), which identifies miRNA and lncRNA interactions derived from manually curated publications and the analysis of 153 AGO CLIP-Seq libraries (15). Data for miRNA and mRNA interactions were downloaded from miRTarBase Release 7.0 (http://mirtarbase. mbc.nctu.edu.tw/php/index.php), which includes data collected via manual survey of the literature following systematic natural language processing of the text to filter research articles, and which are experimentally validated (16).

Construction of the IncRNA-miRNA-mRNA network. Based on the associations between IncRNAs, miRNAs and mRNAs, the ceRNA network was constructed via three steps: i) The upregulated and downregulated RNAs (lncRNAs, miRNAs and mRNAs) were assigned $\mid \log 2$ (fold-change) $\mid>1$ with $\mathrm{P}<0.05$; ii) the 1 cRNAs and mRNAs that were regulated by a certain common miRNA were selected; and iii) the lncRNA-miRNA-mRNA network was constructed and visualized using Cytoscape version 3.5.1 (https://cytoscape.org/), and all node degrees of the ceRNA network were calculated.

Gene Ontology (GO) and pathway enrichment analysis. The GO and Kyoto Encyclopedia of Genes and Genomes (KEGG) analyses of the lncRNA-miRNA-mRNA network were performed using DAVID Bioinformatics Resources 6.8 (https://david.ncifcrf.gov/tools.jsp). DAVID provides a comprehensive set of functional annotation tools to determine the biological relevance of a large set of genes (17). $\mathrm{P}<0.05$ was considered statistically significant.

Participants. A total of 10 patients with HF and 10 age- and sex-matched healthy controls were recruited from The Second Affiliated Hospital of Harbin Medical University (Harbin, China) between December 2017 and January 2018. The diagnosis of HF was made according to the criteria listed in the 2017 ACC/AHA/HFSA Focused Update Guideline for the Management of Heart Failure (18). The inclusion criteria for the patients were: A primary diagnosis of chronic heart failure for at least 3 months; New York Heart Association (NYHA) classification I to III; and, >18 years of age. The diagnosis of $\mathrm{HF}$ was made according to the criteria listed in the 2017 ACC/AHA/HFSA Focused Update Guideline for the Management of Heart Failure (12). The exclusion criteria for the patients included: Myocardial infarction within the previous month, unstable angina, uncontrolled hypertension, severe respiratory disease, decompensated non-cardiac disease, malignancy, physical disability and/or mental disease. The protocols of our study were in accordance with principles of the Helsinki Declaration and Good Clinical Practice and were approved by the Ethics Committee of the Second Affiliated Hospital of Harbin Medical University. The clinical characteristics of the study population are summarized in Table I.

Collection and analysis of human blood samples. Fasting blood $(\sim 5 \mathrm{ml})$ was collected from each participant and anticoagulated with ethylenediaminetetraacetic acid dipotassium salt. The samples were fractionated immediately via centrifugation at $2,000 \times \mathrm{g}$ for $10 \mathrm{~min}$ at $4^{\circ} \mathrm{C}$, and the plasma was stored at $-80^{\circ} \mathrm{C}$ until use. The total cholesterol (TC), total triglyceride (TG), high-density lipoprotein cholesterol (HDL-C), low-density lipoprotein cholesterol (LDL-C) and uric acid (UA) were measured using the Roche enzymatic method (Roche Diagnostics GmbH, Mannheim, Germany). 
Table I. Characteristics of study subjects.

\begin{tabular}{lccc}
\hline Characteristic & Control $(\mathrm{n}=10)$ & HF $(\mathrm{n}=10)$ & P-value \\
\hline Age, years & $69.20 \pm 1.806$ & $67.80 \pm 2.480$ & 0.6536 \\
Sex, F/M & $6 / 4$ & $4 / 6$ & 0.3711 \\
Smoking, $\mathrm{n}(\%)$ & $3(30 \%)$ & $3(30 \%)$ & 1 \\
Alcohol & $3(30 \%)$ & $4(40 \%)$ & 0.6390 \\
consumption, $\mathrm{n}(\%)$ & & & \\
TC, $\mathrm{mmol} / \mathrm{l}$ & $4.210 \pm 0.5291$ & $3.894 \pm 0.3850$ & 0.6350 \\
TG, $\mathrm{mmol} / \mathrm{l}$ & $2.536 \pm 0.7613$ & $1.750 \pm 0.4679$ & 0.3907 \\
HDL-C, mmol/l & $1.121 \pm 0.05417$ & $1.154 \pm 0.1378$ & 0.8261 \\
LDL-C, mmol/ & $2.097 \pm 0.2359$ & $2.463 \pm 0.3403$ & 0.3884 \\
UA, $\mu$ mol/l & $309.4 \pm 20.76$ & $410.7 \pm 45.17$ & 0.0566 \\
NT-proBNP, $\mathrm{pg} / \mathrm{ml}$ & $668.0 \pm 369.3$ & $10,139 \pm 4,224$ & 0.0384 \\
LVEF, $\%$ & $41.10 \pm 0.7219$ & $9(90 \%)$ & $<0.0001$ \\
Hypertension, $\mathrm{n}(\%)$ & $8(80 \%)$ & $4(40 \%)$ & 0.5313 \\
Diabetes, $\mathrm{n}(\%)$ & $3(30 \%)$ & & 0.6390
\end{tabular}

Values are presented as the mean \pm standard deviation unless stated otherwise. $\mathrm{P}<0.05$ was considered indicate a statistically significant difference. TC, total cholesterol; TG, total triglyceride; HDL-C, high-density lipoprotein cholesterol; LDL-C, low-density lipoprotein cholesterol; UA, uric acid; NT-proBNP, N-terminal B-type natriuretic peptide; LVEF, left ventricular ejection fraction; HF, heart failure; F, female; M, male.

N-terminal B-type natriuretic peptide (NT-proBNP) was measured by automated electrochemiluminescence immunoassay method using Elecsys and Cobas analyzers (Roche Diagnostics $\mathrm{GmbH}$ ).

RNA extraction and reverse transcription-quantitative polymerase chain reaction (RT-qPCR). Total RNA was extracted from the plasma samples using TRIzol ${ }^{\circledR}$ reagent (Invitrogen; Thermo Fisher Scientific, Inc., Waltham, MA, USA), as previously described (19). For miRNAs, RT-qPCR was performed using a Hairpin-it microRNA and U6 snRNA Normalization RT-PCR Quantitation kit (Shanghai GenePharma Co., Ltd., Shanghai, China). The conditions for the RT reaction were as follows: $30 \mathrm{~min}$ incubation at $25^{\circ} \mathrm{C}$, $30 \mathrm{~min}$ at $42^{\circ} \mathrm{C}$ and $5 \mathrm{~min}$ at $85^{\circ} \mathrm{C}$. The PCR conditions were $40 \mathrm{cycles}$ of $12 \mathrm{sec}$ at $95^{\circ} \mathrm{C}$ and $40 \mathrm{sec}$ at $62^{\circ} \mathrm{C}$. IncRNA-cDNA transcripts were amplified using a Transcriptor First Strand cDNA Synthesis kit (Roche Diagnostics, Basel, Switzerland), according to the manufacturer's protocol. The RT conditions were as follows: $60 \mathrm{~min}$ at $50^{\circ} \mathrm{C}$ and $5 \mathrm{~min}$ at $85^{\circ} \mathrm{C}$. PCR was performed with Bestar SYBR Green qPCR Master Mix DBI Bioscience (Ludwigshafen, Germany) and the conditions were as follows: 40 cycles of $10 \mathrm{sec}$ at $95^{\circ} \mathrm{C}, 30 \mathrm{sec}$ at $60^{\circ} \mathrm{C}$ and $30 \mathrm{sec}$ at $72^{\circ} \mathrm{C}$. All reactions were performed in triplicate. The expression levels of miRNAs and lncRNAs relative to U6 and $\beta$-actin were respectively determined using the $2^{-\Delta \Delta \mathrm{Cq}}$ method (19). The primers used are listed in Table II.

Statistical analysis. Categorical data are presented as counts and percentiles, and continuous variables as the mean \pm standard deviation. The data were analyzed using GraphPad Prism 7.0 (GraphPad Software, Inc., La Jolla, CA, USA). A two-tailed Student's t-test was used to evaluate the differences between two groups. $\mathrm{P}<0.05$ was considered to indicate a statistically significant difference.

\section{Results}

Screening of DELs, DEMis and DEMs in patients with HF. A total of three gene expression profiles (GSE77399, GSE104150 and GSE84796) were downloaded from the GEO database, and uploaded to GEO2R to screen for the DELs, DEMis and DEMs between HF patients and healthy controls. A total of nine DELs were upregulated and 28 DELs were downregulated in the GSE77399 dataset (Table III). The GSE104150 dataset contained 96 upregulated and 75 downregulated DEMis (data not shown). There were 1,430 upregulated and 835 downregulated DEMs (total, 2,265) in the GSE84796 dataset (data not shown). The differentially expressed transcripts were selected for further analysis.

miRNA predicted target analysis. Target genes of the miRNAs were predicted using experimentally validated databases. The associations between the DELs and DEMis were first assessed using LncBase, which revealed 43 miRNA targets of 12 IncRNAs (Table IV). Subsequently, the associations between these 43 miRNAs and 2,265 DEMs were analyzed using miRTarBase to predict the miRNA targets, and 343 target mRNAs were identified (data not shown). Finally, the 12 co-expressed lncRNAs, 43 co-expressed miRNAs and 343 co-expressed mRNAs were selected to construct the ceRNA network.

lncRNA-miRNA-mRNA network construction. The lncRNA-miRNA-mRNA ceRNA network was constructed using Cytoscape 3.5.1, based on the above data. According to the ceRNA theory, miRNAs negatively regulate the expression 
Table II. List of primers for reverse transcription-quantitative polymerase chain reaction analysis.

\begin{tabular}{lll}
\hline Name & \multicolumn{1}{c}{ Sense sequence $\left(5^{\prime} \rightarrow 3^{\prime}\right)$} & \multicolumn{1}{c}{ Antisense sequence $\left(5^{\prime} \rightarrow 3^{\prime}\right)$} \\
\hline GAS5 & CTTCTGGGCTCAAGTGATCCT & TTGTGCCATGAGACTCCATCAG \\
TUG1 & TAGCAGTTCCCCAATCCTTG & CACAAATTCCCATCATTCCC \\
Hotair & GGTAGAAAAAGCAACCACGAAGC & ACATAAACCTCTGTCTGTGAGTGCC \\
hsa-miR-26b-5p & CGCGGCTTCAAGTAATTCAGG & GTGCAGGGTCCGAGGTATTC \\
hsa-miR-8485 & GCCTCACACACACACACACACGT & GTGCAGGGTCCGAGGT \\
hsa-miR-940 & GCATCGTTCCTTCAAGCCGATCT & TGGGTGAGTCGTTCGG \\
U6 & GTCCTGGCAGATATACACTAAACAT & CTCACGCTTGAATTCATGCGGCTT \\
ACTB & CGTGGACATCCGCAAAGA & GAAGGTGGACAGCGAGGC
\end{tabular}

ACTB, $\beta$-actin; GAS5, growth arrest specific 5; TUG1, taurine upregulated 1; Hotair, HOX transcript antisense RNA; miR, microRNA.

Table III. DELs identified from GSE77399.

DELs Gene names

Upregulated

Downregulated
PCAT-1, GAS5, LINC-ROR, VLDLR-AS1, LINCRNA-SFMBT2, HOTAIR, EGOT, LOC285194, 7SK H19, ANRIL, HOXA11AS, KRASP1, SOX2OT, BC017743, LIT, LUST, RMRP, BACE1AS, PCGEM1, SRA, BC043430, GAPDH2, PGK1, PCAT-14, Y4, ZEB2NAT, NCRMS, TUG1, H19-AS, PAR5,

BC200, UCA1, Y5, IPW, MALAT1, HAR1B

DEL, differentially expressed long noncoding RNA.

Table IV. miRNAs that may target specific lncRNAs.

\begin{tabular}{ll}
\hline IncRNA & \\
\hline EGOT & hsa-miR-183-5p, hsa-miR-491-3p, hsa-miR-586 \\
GAS5 & hsa-let-7a-5p, hsa-let-7g-5p, hsa-let-7i-5p, hsa-miR-101-3p, hsa-miR-15a-3p, hsa-miR-23a-3p, \\
& hsa-miR-26b-5p, hsa-miR-382-3p, hsa-miR-382-3p, hsa-miR-542-3p, hsa-miR-576-5p, \\
& hsa-miR-590-3p, hsa-miR-7f-5p, hsa-miR-876-3p \\
& hsa-miR-491-3p, hsa-miR-548ap-3p, hsa-miR-548c-3p, hsa-miR-548f-3p, hsa-miR-548f-3p, \\
Hotair & hsa-miR-586, hsa-miR-597-5p, hsa-miR-8485 \\
& hsa-miR-1238-3p, hsa-miR-26b-5p, hsa-miR-302d-5p, hsa-miR-580-3p, hsa-miR-616-3p, \\
Linc-ROR & hsa-miR-6883-3p, hsa-miR-8485 \\
PCAT1 & hsa-miR-548c-3p \\
VLDLR-AS1 & hsa-miR-542-3p, hsa-miR-548f-3p \\
H19 & hsa-miR-483-3p, hsa-miR-423-5p, hsa-miR-30d-5p, hsa-miR-940, hsa-miR-324-3p, hsa-miR-1825, \\
& hsa-miR-486-5p \\
HOXA11-AS & hsa-miR-940 \\
SOX2-OT & hsa-miR-574-5p, hsa-miR-320a, hsa-miR-22-3p \\
BACE1-AS & hsa-miR-181a-5p \\
TUG1 & hsa-miR-320c, hsa-miR-320a, hsa-miR-486-5p, hsa-miR-181a-5p, hsa-miR-320b, hsa-miR-1825, \\
BCYRN1 & hsa-miR-1202, hsa-miR-30d-5p, hsa-miR-197-3p, hsa-miR-483-3p, hsa-miR-320d, hsa-miR-574-5p \\
& hsa-miR-22-3p, hsa-miR-320e
\end{tabular}

lncRNA, long noncoding RNA; miRNA, microRNA; GAS5, growth arrest specific 5; TUG1, taurine upregulated 1; Hotair, HOX transcript antisense RNA; EGOT, eosinophil granule ontogeny transcript; Linc-ROR, long intergenic non-protein coding RNA, regulator of reprogramming; PCAT1, prostate cancer associated transcript 1; VLDLR-AS1, VLDLR antisense RNA1; H19, H19, imprinted maternally expressed transcript; HOXA11-AS, HOXA11 antisense RNA; SOX2-OT, SOX2 overlapping transcript; BACE1-AS, BACE1 antisense RNA; BCYRN1, brain cytoplasmic RNA 1. 


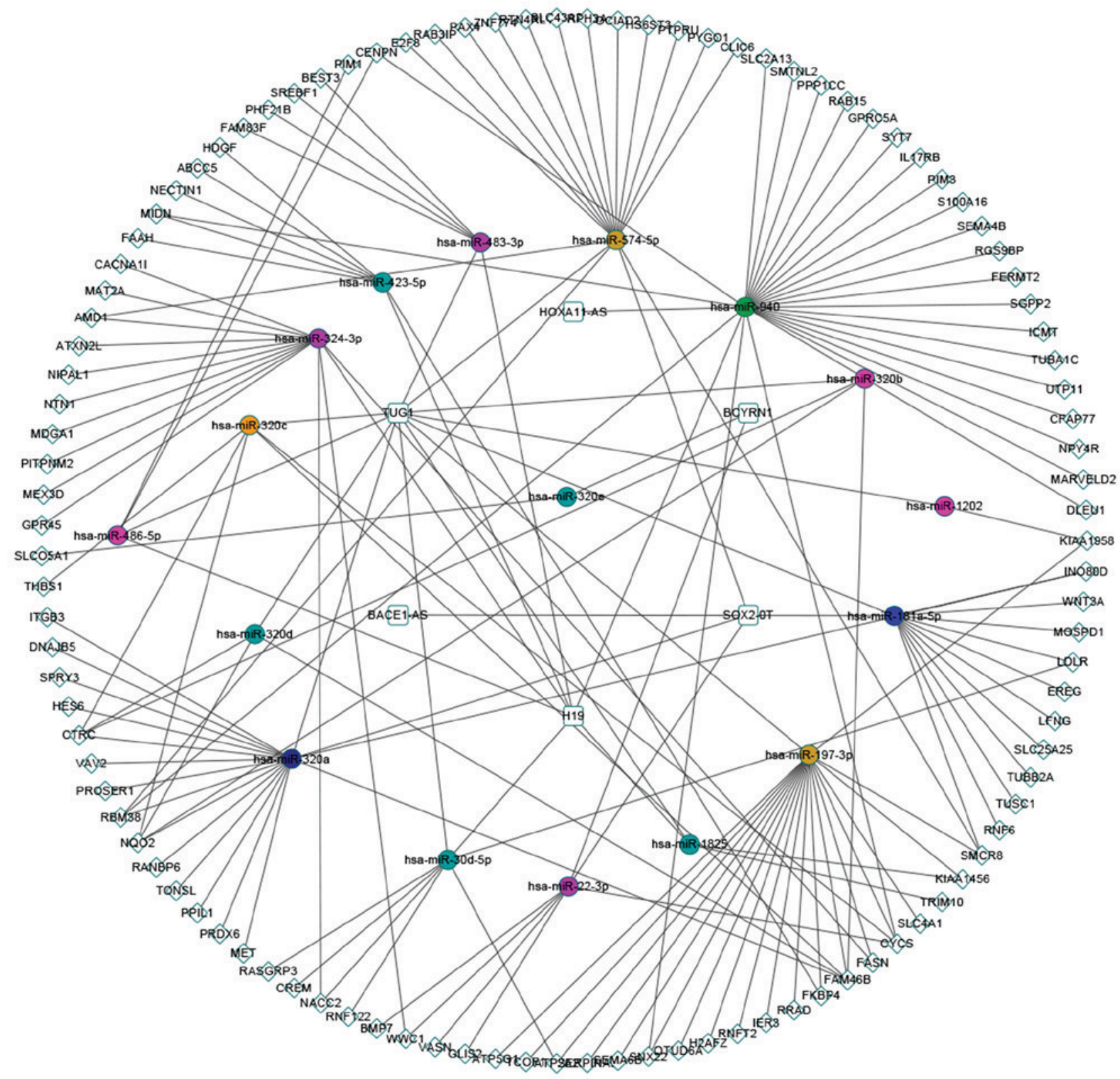

Figure 1. IncRNA-miRNA-mRNA competing endogenous RNA network of upregulated miRNAs. The squares represent lncRNAs, the circles represent miRNAs and the rhombuses represent mRNAs. There were six IncRNA nodes, 17 miRNA nodes and 107 mRNA nodes in the network. miRNA, microRNA; lncRNA, long noncoding RNA.

of lncRNAs and mRNAs. The ceRNA network of upregulated miRNAs is presented in Fig. 1, and consisted of six lncRNA nodes, 17 miRNA nodes, 107 mRNA nodes and 163 edges. The ceRNA network of the downregulated miRNAs (data not shown) included six lncRNA nodes, 26 miRNA nodes, 236 mRNA nodes and 367 edges. The top three ceRNA networks of downregulated miRNAs with high degrees are illustrated in Fig. 2.

Gene ontology enrichment analysis. The function of a ceRNA network depends on the functions of the constituent mRNAs. The biological functions of the DEMs were analyzed using DAVID. GO enrichment analysis of the downregulated miRNA ceRNA network is presented in Fig. 3A. HF is closely associated with immune dysfunction, and numerous GO terms associated with immune responses, including 'adaptive immune response', 'inflammatory response', 'immune response' and 'neutrophil chemotaxis' were obtained. The upregulated miRNA ceRNA network was enriched in terms such as 'protein binding', 'negative regulation of axon extension involved in axon guidance', 'negative chemotaxis' and 'response to peptide hormone' (Fig. 3B). These results indicated that the ceRNA network was primarily associated with immune responses in HF.

KEGG pathway analysis. The initiation and progression of $\mathrm{HF}$ are associated with alterations in a number of biological pathways. Therefore, a pathway enrichment analysis was performed on the ceRNA networks using KEGG. The downregulated miRNA ceRNA network was primarily enriched in the pathways 'natural killer cell mediated cytotoxicity', 'T cell receptor signaling pathway' and 'B cell receptor signaling 

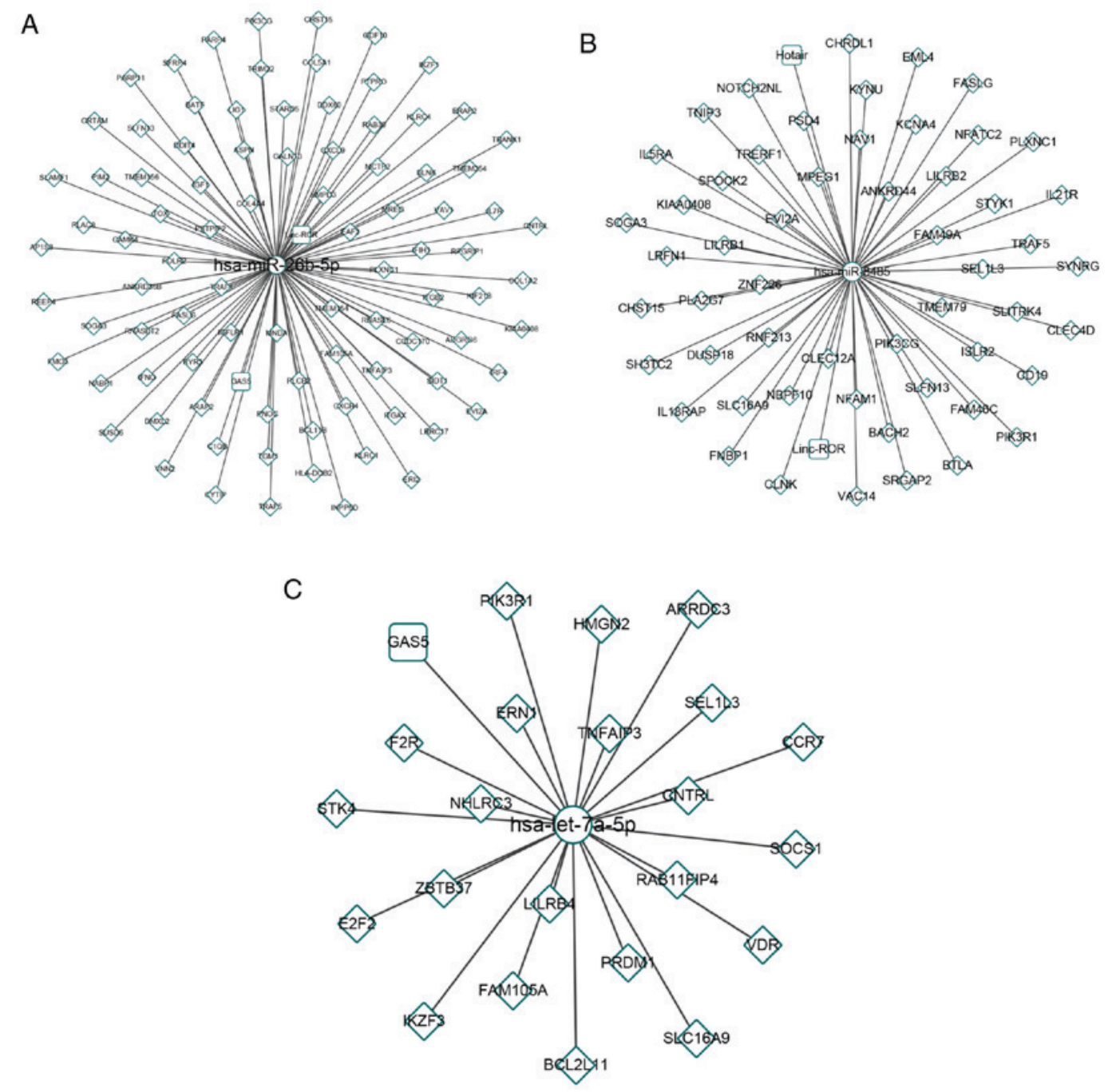

Figure 2. Top three miRNAs ranked by node degree. The sub-networks of (A) hsa-miR-26b-5p, (B) hsa-miR-8485 and (C) hsa-let-7a-5p are presented. The circles represent miRNAs, the squares represent long noncoding RNAs and the rhombuses represent mRNAs. miR/miRNA, microRNA.

A

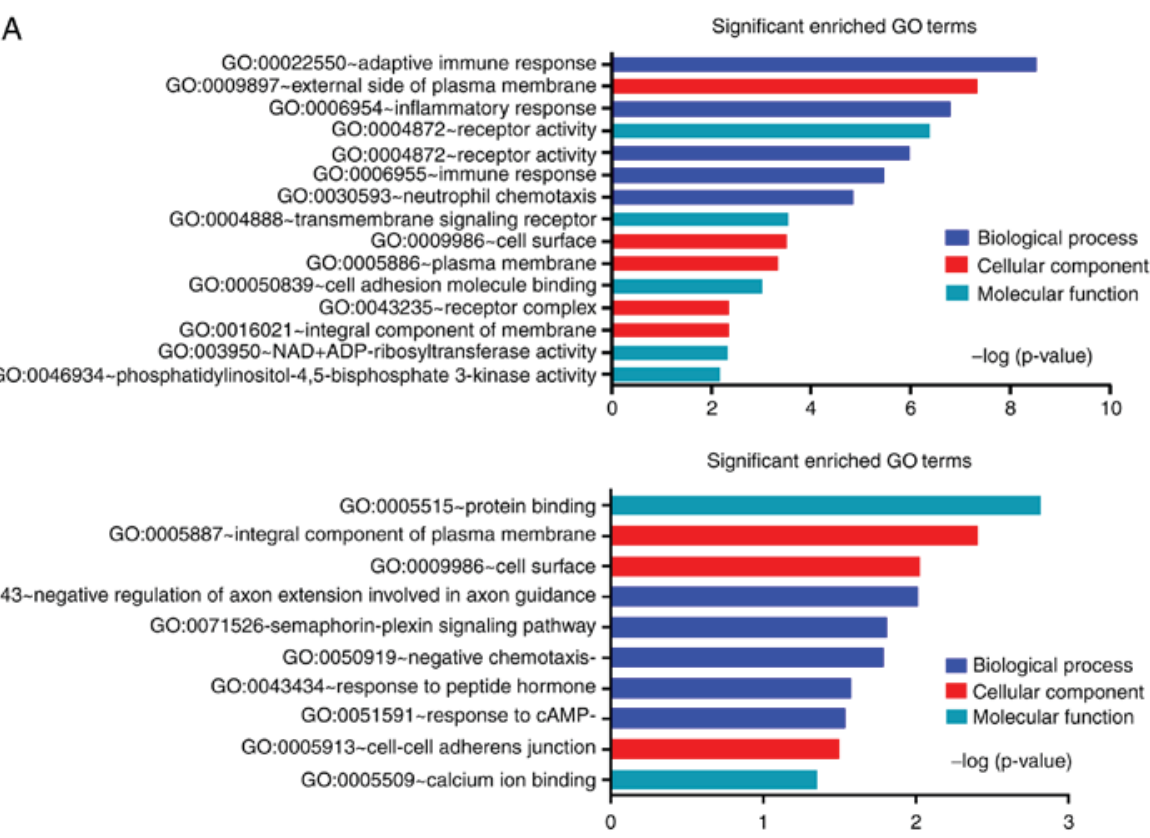

B

Figure 3. GO enrichment analysis. (A) Downregulated miRNA ceRNA network. (B) Upregulated miRNA ceRNA network. GO analysis classified the differentially expressed genes into three groups, including biological process, cellular component and molecular function. GO, gene ontology; miRNA, microRNA; ceRNA, competing endogenous RNA. 


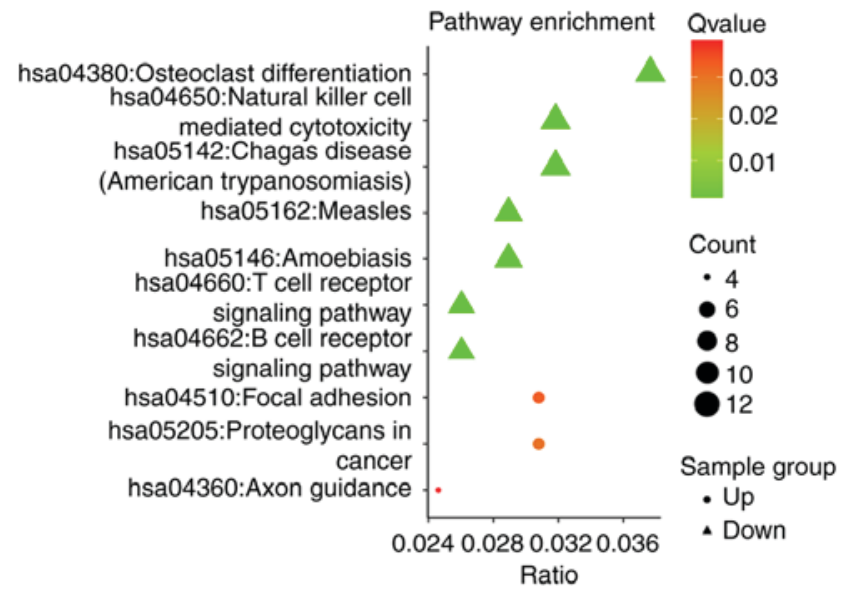

Figure 4. Kyoto Encyclopedia of Genes and Genomes pathway analysis. The triangles represent the downregulated miRNA ceRNA network and the circles represent the upregulated miRNA ceRNA network. miRNA, microRNA; ceRNA, competing endogenous RNA.

pathway' (Fig. 4). The upregulated miRNA ceRNA network was significantly enriched in the 'focal adhesion', 'axon guidance' and 'proteoglycans in cancer' pathways. KEGG analysis revealed that the ceRNA network in HF likely alters the pathways of immune regulation.

Topological analysis of the HF-associated ceRNA network. It is well known that hub nodes serve key roles in biological networks. Therefore, all the node degrees of the ceRNA network were calculated using Cytoscape. It has previously been reported that the degree of a hub node should exceed 5 (20). Based on this criterion, 38 nodes were identified as hub nodes, including five lncRNAs, 31 miRNAs, and two mRNAs (Table V). hsa-miR-26b-5p, hsa-miR-8485 and hsa-miR-940 were the top three hub miRNAs in the ceRNA network (Table V), and GAS5, TUG1 and Hotair had higher degrees compared with the other lncRNAs. These top hub nodes may serve a crucial role in the genesis and development of HF.

Validation of hub nodes in patients with HF. To further validate the importance of the hub nodes in HF, 10 patients with $\mathrm{HF}$ and 10 matched controls were enrolled to test for the serum levels of the different transcripts. The characteristics of the study population are presented in Table I. No significant differences were identified in terms of age, gender, smoking, alcohol consumption, TC, TG, HDL-C, LDL-C, UA, hypertension and diabetic status. Previous studies have demonstrated that HF-associated lncRNAs may be detected in heart tissue and plasma $(21,22)$. The patients with HF had higher BNP $(10,139 \pm 4,224$ vs. $668.0 \pm 369.3)$ and lower LVEF (42.40 \pm 2.561 vs. $61.10 \pm 0.7219)$ levels compared to the controls. In addition, the levels of hsa-miR-940, GAS5 and Hotair were higher in the patients with HF compared with the controls (Fig. 5). However, the circulating levels of hsa-miR-26-5p, hsa-miR-8485 and TUG1 were lower in patients with HF compared with the matched controls (Fig. 5). Taken together, the expression of the hub nodes (GAS5, TUG1, Hotair, hsa-miR-26b-5p, hsa-miR-8485 and hsa-miR-940) was significantly altered in patients with HF,
Table V. List of differentially expressed genes (node degree $>5$ ).

\begin{tabular}{|c|c|c|}
\hline \multicolumn{3}{|l|}{ A, miRNA } \\
\hline Gene name & Node degree & Direction of regulation \\
\hline hsa-miR-26b-5p & 92 & Down \\
\hline hsa-miR-8485 & 56 & Down \\
\hline hsa-miR-940 & 27 & Up \\
\hline hsa-let-7a-5p & 23 & Down \\
\hline hsa-miR-548c-3p & 22 & Down \\
\hline hsa-miR-197-3p & 20 & Up \\
\hline hsa-miR-320a & 18 & Up \\
\hline hsa-miR-574-5p & 17 & $\mathrm{Up}$ \\
\hline hsa-miR-183-5p & 16 & Down \\
\hline hsa-miR-101-3p & 14 & Down \\
\hline hsa-miR-324-3p & 14 & Up \\
\hline hsa-miR-181a-5p & 13 & Up \\
\hline hsa-miR-548f-3p & 12 & Down \\
\hline hsa-miR-7f-5p & 12 & Down \\
\hline hsa-miR-590-3p & 12 & Down \\
\hline hsa-miR-23a-3p & 12 & Down \\
\hline hsa-let-7g-5p & 11 & Down \\
\hline hsa-let-7i-5p & 10 & Down \\
\hline hsa-miR-616-3p & 8 & Down \\
\hline hsa-miR-548ap-3p & 8 & Down \\
\hline hsa-miR-30d-5p & 8 & Up \\
\hline hsa-miR-580-3p & 7 & Down \\
\hline hsa-miR-542-3p & 7 & Down \\
\hline hsa-miR-491-3p & 7 & Down \\
\hline hsa-miR-22-3p & 7 & Up \\
\hline hsa-miR-423-5p & 7 & Up \\
\hline hsa-miR-302d-5p & 6 & Down \\
\hline hsa-miR-1238-3p & 6 & Down \\
\hline hsa-miR-586 & 6 & Down \\
\hline hsa-miR-320c & 6 & Up \\
\hline hsa-miR-483-3p & 6 & Up \\
\hline
\end{tabular}

\section{$\mathrm{B}, \operatorname{lncRNA}$}

\begin{tabular}{lcc}
\hline Gene name & Node degree & Direction of regulation \\
\hline GAS5 & 14 & Up \\
TUG1 & 12 & Down \\
Hotair & 8 & Up \\
Linc-ROR & 7 & Up \\
H19 & 7 & Down \\
\hline
\end{tabular}

\section{C, mRNA}

\begin{tabular}{lcc}
\hline Gene name & Node degree & Direction of regulation \\
\hline HMGN2 & 6 & Up \\
SLC16A9 & 6 & Up \\
\hline
\end{tabular}

lncRNA, long non-coding RNA; GAS5, growth arrest specific 5; TUG1, taurine upregulated 1; Hotair, HOX transcript antisense RNA; Linc-ROR, long intergenic non-protein coding RNA, regulator of reprogramming; H19, H19, imprinted maternally expressed transcript; HMGN2, high mobility group nucleosomal binding domain 2; SLC16A9, solute carrier family 16 member 9. 

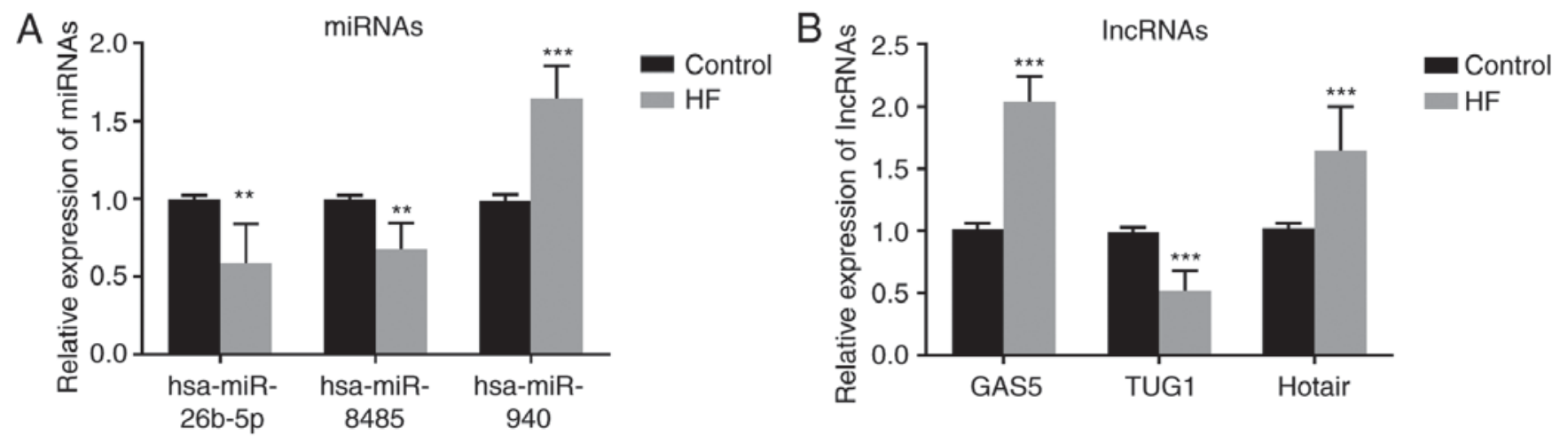

Figure 5. Hub nodes detection by reverse transcription-quantitative polymerase chain reaction. (A) The relative expression of hsa-miR-26b-5p and hsa-miR-8485 was decreased, and hsa-miR-940 was increased, in patients with HF. (B) The relative expression of GAS5 and Hotair was, increased and TUG1 was decreased, in patients with HF. The data indicate the mean \pm standard deviation of triplicate samples, from three independent experiments. ${ }^{* *} \mathrm{P}<0.01,{ }^{* * *} \mathrm{P}<0.001 \mathrm{vs}$. respective control group. HF, heart failure; miRNA/miR, microRNA; lncRNA, long noncoding RNA; GAS5, growth arrest specific 5; TUG1, taurine upregulated 1; Hotair, HOX transcript antisense RNA.

and thus they may serve as biomarkers and therapeutic targets for HF. However, due the small size of the cohort, the clinical roles and biological functions of these hub genes require further validation with more experiments.

\section{Discussion}

HF is a chronic disease with high morbidity and mortality worldwide, which results in an increased economic burden for patients and their families (23). Although numerous studies have been conducted on the molecular mechanisms of HF, the pathogenesis of $\mathrm{HF}$ is not well understood and requires further research $(23,24)$. Previous studies have largely focused on protein-coding genes (25). Recently however, with the development of high-throughput genomic platforms, noncoding RNAs, including miRNAs and lncRNAs, have gained more attention. They serve an important role in the regulation of multiple biological processes, including development, differentiation and metabolism $(25,26)$. ceRNA is a common regulatory mechanism used by miRNAs and IncRNAs (11), and studies have demonstrated its involvement in various pathogeneses, including cancer (27-29). However, the ceRNA network in HF has not been systematically examined. In the present study, HF-associated gene expression datasets were downloaded from the GEO to construct the ceRNA network.

The present study identified the differentially expressed transcripts from GSE77399, GSE104150 and GSE84796, and predicted the target lncRNAs and mRNAs of miRNAs. Although the datasets were from different HF patients, as per previous studies, the differentially expressed genes could be analyzed for constructing an lncRNA-miRNA-mRNA ceRNA network $(30,31)$. The ceRNA network of up-regulated miRNAs consisted of six lncRNA nodes, 17 miRNA nodes, 107 mRNA nodes and 163 edges. The ceRNA network of downregulated miRNAs consisted of six lncRNA nodes, 26 miRNA nodes, 236 mRNA nodes and 367 edges. GO analysis and KEGG pathway analysis demonstrated that the function of the ceRNA network was associated with immune regulation. The most significant biological process categories of GO terms were 'adaptive immune response', 'inflammatory response', 'immune response', 'negative chemotaxis' and 'response to peptide hormone', while the KEGG analysis demonstrated enrichment in 'natural killer cell mediated cytotoxicity', 'T cell receptor signaling pathway', 'B cell receptor signaling pathway' and 'focal adhesion'. These results are consistent with previous studies that have demonstrated that activation of the immune response contributes to the development of $\mathrm{HF}$ with reduced or preserved ejection function $(32,33)$. In order to identify the key genes that may be used as potential diagnostic biomarkers and therapeutic targets of HF, the hub nodes were calculated. Hub nodes with high degree of connectivity to other nodes may be used as topological markers of the ceRNA network to evaluate the important genes (34). The top three miRNAs (hsa-miR-26b-5p, hsa-miR-8485 and hsa-miR-940) and three lncRNAs (GAS5, TUG1 and Hotair) were identified as the key hub nodes whose degrees were significantly higher compared with the other nodes. These hub genes were verified in patients with HF by RT-qPCR. Reduced levels of hsa-miR-26b-5p have been associated with increasing severity of HF (35), and a significant correlation was observed between hsa-miR-26b-5p and left ventricular end-systolic pressure and $\mathrm{dP} / \mathrm{dt}_{\max }$ values (36). hsa-miR-940 is enriched in human cardiac tissues (37), and is upregulated in patients with HF in response to acute exercise (38). Therefore, hsa-miR-940 may have beneficial effects in HF. hsa-miR-8485 has recently been demonstrated to bind to TDP-43, although limited knowledge is available as to its role in HF (38).

Cardiac fibrosis is an important characteristic of myocardial structural remodeling in HF (39). GAS5 is expressed at low levels in fibrotic cardiac tissue and serves a suppressive role in HF via negative regulation of miR-21 (40). Hotair is upregulated and may be a biomarker in patients with HF (41). Furthermore, a mechanistic study demonstrated that Hotair modulated phosphatase and tensin homolog expression in $\mathrm{HF}$ by competing with miR-19 (42). A previous study demonstrated the important role of TUG1 in coronary heart disease (43). These findings indicate that these miRNAs and lncRNAs may have profound implications for $\mathrm{HF}$, and thus may be considered to be key regulatory genes.

In conclusion, based on the ceRNA hypothesis, a lncRNA-miRNA-mRNA ceRNA network was constructed for 
HF. The present findings indicated that the ceRNA networks may serve important roles in the development of $\mathrm{HF}$, and were enriched in immune response functions. hsa-miR-26b-5p, hsa-miR-8485, hsa-miR-940, GAS5, TUG1 and Hotair were identified as the key genes. The present study increased the understanding of the ceRNA-associated regulatory mechanism in $\mathrm{HF}$, and helped to identify potential diagnostic biomarkers or therapeutic targets in HF.

\section{Acknowledgements}

The authors would like to thank Dr Yuanyuan Wei (China Pharmaceutical University, Jiangsu, China) for technical support and critical review of the manuscript.

\section{Funding}

This study was supported by the National Natural Science Foundation of China (grant nos. 81670373, 81670459 and 81771946).

\section{Availability of data and materials}

The datasets used and/or analyzed during the current study are available from the corresponding author on reasonable request.

\section{Authors' contributions}

GW and $\mathrm{XZ}$ conceived and designed the study. YZ and RC performed the experiments. MZ, YS and JW analyzed the data. GW and XZ wrote the paper. YS and JW revised the manuscript and have given final approval of the version to be published.

\section{Ethics approval and consent to participate}

Human peripheral blood was isolated from patients with heart failure and healthy volunteers with their written informed consent. The project was approved by the Clinical Research Ethics Committee of The Second Affiliated Hospital of Harbin Medical University (Harbin, China).

\section{Patient consent for publication}

Not applicable.

\section{Competing interests}

The authors declare that they have no competing interests.

\section{References}

1. Braunwald E: The war against heart failure: The Lancet lecture. Lancet 385: 812-824, 2015 .

2. Tham YK, Bernardo BC, Ooi JY, Weeks KL and McMullen JR: Pathophysiology of cardiac hypertrophy and heart failure: Signaling pathways and novel therapeutic targets. Arch Toxicol 89: 1401-1438, 2015.

3. Moran AE, Forouzanfar MH, Roth GA, Mensah GA, Ezzati M, Flaxman A, Murray CJ and Naghavi M: The global burden of ischemic heart disease in 1990 and 2010: The Global burden of disease 2010 study. Circulation 129: 1493-1501, 2014.

4. Tanai E and Frantz S: Pathophysiology of heart failure. Compr Physiol 6: 187-214, 2015.
5. Kovács Á, Papp Z and Nagy L: Causes and pathophysiology of heart failure with preserved ejection fraction. Heart Fail Clin 10: 389-398, 2014.

6. Zhang Y, Bauersachs J and Langer HF: Immune mechanisms in heart failure. Eur J Heart Fail 19: 1379-1389, 2017.

7. Hulot JS, Ishikawa K and Hajjar RJ: Gene therapy for the treatment of heart failure: Promise postponed. Eur Heart J 37: 1651-1658, 2016.

8. Xu S, Kamato D, Little PJ, Nakagawa S, Pelisek J and Jin ZG: Targeting epigenetics and non-coding RNAs in atherosclerosis: From mechanisms to therapeutics. Pharmacol Ther: Nov 12, 2018 (Epub ahead of print).

9. Wong CM, Tsang FH and Ng IO: Non-coding RNAs in hepatocellular carcinoma: Molecular functions and pathological implications. Nat Rev Gastroenterol Hepatol 15: 137-151, 2018.

10. Sallam T, Sandhu J and Tontonoz P: Long noncoding RNA discovery in cardiovascular disease: Decoding form to function. Circ Res 122: 155-166, 2018.

11. Salmena L, Poliseno L, Tay Y, Kats L and Pandolfi PP: A ceRNA hypothesis: The rosetta stone of a hidden RNA language? Cell 146: 353-358, 2011.

12. Chen X, Chen Z, Yu S, Nie F, Yan S, Ma P, Chen Q, Wei C, $\mathrm{Fu} \mathrm{H}, \mathrm{Xu}$ T, et al: Long noncoding RNA LINC01234 functions as a competing endogenous RNA to regulate CBFB expression by sponging miR-204-5p in gastric cancer. Clin Cancer Res 24: 2002-2014, 2018.

13. Barrett T, Wilhite SE, Ledoux P, Evangelista C, Kim IF, Tomashevsky M, Marshall KA, Phillippy KH, Sherman PM, Holko M, et al: NCBI GEO: Archive for functional genomics data sets-update.Nucleic AcidsRes41 (DatabaseIssue):D991-D995,2013.

14. Zheng Y, Zheng X, Li S, Zhang H, Liu M, Yang Q, Zhang M, Sun Y, Wu J and Yu B: Identification of key genes and pathways in regulating immune-induced diseases of dendritic cells by bioinformatic analysis. Mol Med Rep 17: 7585-7594, 2018.

15. Paraskevopoulou MD, Vlachos IS, Karagkouni D, Georgakilas G, Kanellos I, Vergoulis T, Zagganas K, Tsanakas P, Floros E, Dalamagas T and Hatzigeorgiou AG: DIANA-LncBase v2: Indexing microRNA targets on non-coding transcripts. Nucleic Acids Res 44 (D1): D231-D238, 2016.

16. Chou CH, Shrestha S, Yang CD, Chang NW, Lin YL, Liao KW, Huang WC, Sun TH, Tu SJ, Lee WH, et al: miRTarBase update 2018: A resource for experimentally validated microRNA-target interactions. Nucleic Acids Res 46 (D1): D296-D302, 2018.

17. Huang da W, Sherman BT and Lempicki RA: Systematic and integrative analysis of large gene lists using DAVID bioinformatics resources. Nat Protoc 4: 44-57, 2009.

18. Yancy CW, Jessup M, Bozkurt B, Butler J, Casey DE Jr, Colvin MM, Drazner MH, Filippatos GS, Fonarow GC, Givertz MM, et al: 2017 ACC/AHA/HFSA focused update of the 2013 ACCF/AHA guideline for the management of heart failure: A report of the American college of cardiology/American heart association task force on clinical practice guidelines and the heart failure society of America. J Am Coll Cardiol 70: 776-803, 2017.

19. Zhang M, Liu F, Jia H, Zhang Q, Yin L, Liu W, Li H, Yu B and Wu J: Inhibition of microRNA let-7i depresses maturation and functional state of dendritic cells in response to lipopolysaccharide stimulation via targeting suppressor of cytokine signaling 1 . J Immunol 187: 1674-1683, 2011.

20. Han JD, Bertin N, Hao T, Goldberg DS, Berriz GF, Zhang LV, Dupuy D, Walhout AJ, Cusick ME, Roth FP and Vidal M: Evidence for dynamically organized modularity in the yeast protein-protein interaction network. Nature 430: 88-93, 2004.

21. Gao L, Liu Y, Guo S, Yao R, Wu L, Xiao L, Wang Z, Liu Y and Zhang Y: Circulating long noncoding RNA HOTAIR is an essential mediator of acute myocardial infarction. Cell Physiol Biochem 44: 1497-1508, 2017.

22. Greco S, Zaccagnini G, Fuschi P, Voellenkle C, Carrara M, Sadeghi I, Bearzi C, Maimone B, Castelvecchio S, Stellos K, et al: Increased BACE1-AS long noncoding RNA and $\beta$-amyloid levels in heart failure. Cardiovasc Res 113: 453-463, 2017.

23. O'Connor CM, Psotka MA, Fiuzat M, Lindenfeld J, Abraham WT, Bristow MR, Canos D, Harrington RA, Hillebrenner M, Jessup M, et al: Improving heart failure therapeutics development in the United States: The heart failure collaboratory. J Am Coll Cardiol 71: 443-453, 2018.

24. Pearson MJ, Mungovan SF and Smart NA: Effect of aerobic and resistance training on inflammatory markers in heart failure patients: Systematic review and meta-analysis. Heart Fail Rev 23: 209-223, 2018. 
25. Wang KC and Chang HY: Molecular mechanisms of long noncoding RNAs. Mol Cell 43: 904-914, 2011.

26. Ulitsky I and Bartel DP: lincRNAs: Genomics, evolution, and mechanisms. Cell 154: 26-46, 2013.

27. Shuwen H, Qing Z, Yan Z and Xi Y: Competitive endogenous RNA in colorectal cancer: A systematic review. Gene 645: 157-162, 2018.

28. Yang C, Wu D, Gao L, Liu X, Jin Y, Wang D, Wang T and Li X: Competing endogenous RNA networks in human cancer: Hypothesis, validation, and perspectives. Oncotarget 7: 13479-13490, 2016.

29. Qi X, Zhang DH, Wu N, Xiao JH, Wang X and Ma W: ceRNA in cancer: Possible functions and clinical implications. J Med Genet 52: 710-718, 2015.

30. Jiang H, Ma R, Zou S, Wang Y, Li Z and Li W: Reconstruction and analysis of the 1ncRNA-miRNA-mRNA network based on competitive endogenous RNA reveal functional lncRNAs in rheumatoid arthritis. Mol Biosyst 13: 1182-1192, 2017.

31. Pang L, Hu J, Zhang G, Li X, Zhang X, Yu F, Lan Y, Xu J, Pang B, Han D, et al: Dysregulated long intergenic non-coding RNA modules contribute to heart failure. Oncotarget 7: 59676-59690, 2016.

32. Dick SA and Epelman S: Chronic heart failure and inflammation: What do we really know? Circ Res 119: 159-176, 2016.

33. Fildes JE, Shaw SM, Yonan N and Williams SG: The immune system and chronic heart failure: Is the heart in control? J Am Coll Cardiol 53: 1013-1020, 2009.

34. Browne AL, Charmsaz S, Varešlija D, Fagan A, Cosgrove N Cocchiglia S, Purcell S, Ward E, Bane F, Hudson L, et al: Network analysis of SRC-1 reveals a novel transcription factor hub which regulates endocrine resistant breast cancer. Oncogene 37: 2008-2021, 2018.

35. Ovchinnikova ES, Schmitter D, Vegter EL, Ter Maaten JM, Valente MA, Liu LC, van der Harst P, Pinto YM, de Boer RA, Meyer S, et al: Signature of circulating microRNAs in patients with acute heart failure. Eur J Heart Fail 18: 414-423, 2016.
36. Vegter EL, Ovchinnikova ES, Silljé HHW, Meems LMG, van der Pol A, van der Velde AR, Berezikov E, Voors AA, de Boer RA and van der Meer P: Rodent heart failure models do not reflect the human circulating microRNA signature in heart failure. PLoS One 12: e0177242, 2017.

37. Liang D, Xu X, Deng F, Feng J, Zhang H, Liu Y, Zhang Y, Pan L, Liu Y, Zhang D, et al: miRNA-940 reduction contributes to human tetralogy of fallot development. J Cell Mol Med 18: 1830-1839, 2014.

38. Xu T, Zhou Q, Che L, Das S, Wang L, Jiang J, Li G, Xu J, Yao J, Wang $\mathrm{H}$, et al: Circulating miR-21, miR-378, and miR-940 increase in response to an acute exhaustive exercise in chronic heart failure patients. Oncotarget 7: 12414-12425, 2016.

39. Nevers T, Salvador AM, Velazquez F, Ngwenyama N, Carrillo-Salinas FJ, Aronovitz M, Blanton RM and Alcaide P: Th1 effector T cells selectively orchestrate cardiac fibrosis in nonischemic heart failure. J Exp Med 214: 3311-3329, 2017.

40. Tao H, Zhang JG, Qin RH, Dai C, Shi P, Yang JJ, Deng ZY and Shi KH: LncRNA GAS5 controls cardiac fibroblast activation and fibrosis by targeting miR-21 via PTEN/MMP-2 signaling pathway. Toxicology 386: 11-18, 2017.

41. Greco S, Zaccagnini G, Perfetti A, Fuschi P, Valaperta R, Voellenkle C, Castelvecchio S, Gaetano C, Finato N, Beltrami AP, et al: Long noncoding RNA dysregulation in ischemic heart failure. J Transl Med 14: 183, 2016.

42. Lai Y, He S, Ma L, Lin H, Ren B, Ma J, Zhu X and Zhuang S: HOTAIR functions as a competing endogenous RNA to regulate PTEN expression by inhibiting miR-19 in cardiac hypertrophy. Mol Cell Biochem 432: 179-187, 2017.

43. Wu Z, Zhao S, Li C and Liu C: LncRNA TUG1 serves an important role in hypoxia-induced myocardial cell injury by regulating the miR-145-5p-Binp3 axis. Mol Med Rep 17: 2422-2430, 2018.

(i) $(5)$ This work is licensed under a Creative Commons Attribution-NonCommercial-NoDerivatives 4.0 International (CC BY-NC-ND 4.0) License. 